\title{
On the dynamics and shapes of star clusters
}

\author{
William H. Jefferys \\ University of Texas at Austin, Austin, Texas \\ (Received 28 June 1976; revised 13 August 1976)
}

\begin{abstract}
The dynamics of a simplified star cluster, perturbed by the galaxy, have been investigated by the method of surface of section. In agreement with Keenan, Innanen, and House (1973), it is found that retrograde orbits moving around the cluster in a direction opposite to the motion of the cluster around the galaxy are much more stable than direct orbits moving in the same sense as the motion of the cluster as a whole, and this is attributed to the existence of a second quasiintegral in addition to the energy integral. The shape of the cluster is strongly affected by galactic tides. Another model, for clusters moving in rectilinear orbits, exhibits a similar effect of galactic tides on the cluster shape. Observed shapes of clusters are compared with the predictions of the theoretical models. Agreement with observations is found in some cases, but not in all.
\end{abstract}

\section{INTRODUCTION}

$\mathbf{I}^{\mathrm{T}}$ IS known (Shapley 1930) that many globular clusters are significantly flattened, and the same appears to be true of galactic clusters, as predicted by Jeans (1922) in work to be discussed later in this paper. The conclusion in the case of galactic clusters is less secure, due to their relative poorness. Shapley found no correlation between the orientation of the projected long axes of globulars and the galactic equator, and little correlation for galactic clusters, although certain longitudes appeared to exhibit such correlation. On the other hand, Johnson (1952) found that Shapley's data on globular clusters were consistent with the hypothesis that the equatorial planes of globular clusters pass through the galactic center. He suggested that this was due to a distortion in the shape of the cluster which persists after a cluster's perigalactic passage. Keenan, Innanen, and House (1973) studied the orbits of individual stars in globular clusters orbiting the galaxy. They found that retrograde orbits (those traveling around the cluster in a sense opposite to the cluster's sense of revolution around the galaxy) were much more stable than direct orbits, and suggested that the perigalactic passages of a cluster would tend to "prune away" the direct orbits, leaving the cluster with a net retrograde rotation. Rotation has been observed by Harding (1965) in $\omega$ Cen (NGC 5139), and by Griffin (1972) in M3 (NGC 6205).

Keenan and Innanen (1975), noting work by Jackson (1913), Hénon (1969a, 1970), Hunter (1967a, 1967b) and Jefferys (1974), mainly in connection with the restricted three-body problem, found certain combinations of energy and angular momentum which are avoided by stars escaping a cluster, and speculated that "quasiintegrals" of motion analogous to the Jacobi integral of the restricted three-body problem may also operate in the case of clusters. However, they did not consider the possible existence of a pseudointegral which would bind the orbits to a region smaller than that which would be allowed by a Jacobi-type integral.
In another study, Hopper and Disney (1974), using Lynds' (1962) data, found that interstellar dust clouds also exhibit a significant degree of alignment with the galactic plane; they explained this as an effect due to the differential gravitational field of the galaxy perpendicular to the plane, which would tend to flatten the cloud out. Significantly, they noted that the tendency for alignment was especially strong near $l^{\mathrm{II}}=90^{\circ}$ (the opposite direction was not included in Lynds' catalog).

Finally, Melnick and Harwit (1975) have noted a similar and statistically significant alignment of the orientations of planetary nebulae with the galactic plane. They offered no explanation for this effect.

\section{A SIMPLIFIED CLUSTER MODEL}

To study the possible existence of pseudointegrals in star clusters, a simplified model has been investigated. If a potential center with potential $\Phi$ (the cluster) moves in a circular orbit around the galaxy, the equations of motion which govern a star moving under the influence of both the potential center and the galaxy can be approximated by a modified Hill's problem:

$$
\begin{aligned}
\ddot{x}-2 n \dot{y}-\alpha n^{2} x & =\partial \Phi / \partial x, \\
\ddot{y}+2 n \dot{x} & =\partial \Phi / \partial y, \\
\ddot{z} & =\partial \Phi / \partial z .
\end{aligned}
$$

Here, $n$ is the (constant) angular velocity of the cluster around the galaxy, the $x, y$ plane is in the orbital plane of the cluster, and the $x$ axis points towards the center of the galaxy. $\alpha$ is a constant of order unity characteristic of the force law at the given distance from the galactic center. For a point mass (considered here) $\alpha=3$. Expressed in terms of Oort's constants, it can be shown that $\alpha=4 A /(A-B) \approx 2.4$ for the solar neighborhood, so the approximation of a point-mass galaxy is a good one. The coordinates are therefore rotating coordinates centered on the cluster. An important approximation made here is that the orbit of the cluster is circular. This is unreal- 
istic for globulars, but is certainly reasonable for galactic clusters.

If the cluster were not in a circular orbit, then the Coriolis terms proportional to $\dot{x}$ and $\dot{y}$ would vary with time, as would the galactic tidal force terms linear in $x$. However, this would complicate the model so as to make interpretation much more difficult, and has not been attempted here.

If the galaxy were a point mass, we would have

$$
n^{2} A^{3}=G(M+m),
$$

where $A$ is the distance of the cluster from the galactic center, $M$ is the mass of the galaxy, $m$ is the mass of the cluster, and $G$ is the constant of gravity. Even though the galaxy is not a point mass, this relation will be satisfied as to order of magnitude.

Equations (1) possess an integral, the Jacobi integral:

$$
\left(\dot{x}^{2}+\dot{y}^{2}+\dot{z}^{2}\right)-\alpha n^{2} x^{2}-2 \Phi=C,
$$

where $C$ is the Jacobi constant. However, if the orbit of the cluster were elliptical, this integral would not exist. In the integrations to be described, $C$ becomes a fundamental parameter.

$\Phi$ has been approximated with a Plummer potential (polytrope of index 5). According to Hénon (1969b) we have

$$
\Phi=G m /\left(r^{2}+r_{0}^{2}\right)^{1 / 2},
$$

where $r^{2}=x^{2}+y^{2}+z^{2}$, and $r_{0}$ is a scale height or typical cluster radius. While this is not the most realistic possible potential (see King 1962), it is easy to work with and is a sufficiently good approximation for this very simple model, since, as will be seen, tidal distortions seriously affect the outer regions of the cluster, certainly more so than the errors introduced by the use of this potential.

Adopting natural units in which $r_{0}=m=G=1$, and picking a point-mass galaxy, the only adjustable parameter in the equations becomes $n$, which may be thought of as the ratio of the period of the cluster around the galaxy to the period a star around the cluster. To estimate $n$ for typical cases, we might have for a galactic cluster in the solar neighborhood, $M \sim 10^{11} M_{\odot}, m \sim$ $10^{2} M_{\odot}, A \sim 10^{4} \mathrm{pc}, r_{0} \sim 5 \mathrm{pc}$, and $n \sim 0.35$; for a globular cluster, we might have $M \sim 2 \times 10^{11} M_{\odot}, m \sim$ $5 \times 10^{5} M_{\odot}, A \sim 10^{3} \mathrm{pc}, r_{0} \sim 5 \mathrm{pc}$, and $n \sim 0.2$. Thus, values of $n$ of order $0.2-0.4$ are quite reasonable. More compact clusters (for example, the Plieades) have somewhat smaller values of $n \sim 0.1-0.2$.

A "theoretical" value of $n$ could be estimated as follows: Assuming the cluster to be tidally limited, its radius $r_{0}=1$ (in natural units) would coincide with the opening of the Roche lobe of the equipotential surface at $x_{0}=1$, $y_{0}=0$. At this point the gradient of the combined galactic and cluster potential vanishes, so that

$$
-\frac{x_{0}}{\left(1+x_{0}\right)^{3 / 2}}+\alpha n^{2} x_{0}=0
$$

or, solving for $n$ when $x_{0}=1$,

$$
n=8^{-1 / 4} \alpha^{-1 / 2} \approx 0.3433 \text { for } \alpha=3 \text {. }
$$

This value was actually adopted for the integrations described here, for it is consistent with the values typical of real clusters, and has a certain theoretical appeal.

Another way of looking at the value of $n$ to be adopted is to consider the linearization of Eqs. (1). We have then

$$
\nabla \Phi=-\frac{\hat{r}}{\left(1+r^{2}\right)^{3 / 2}} \approx-\hat{r},
$$

where $\hat{r}$ is the unit vector from the origin to the star. The $z$ equation becomes a harmonic oscillator decoupled from the $x, y$ equations. The $x$ and $y$ equations are

$$
\begin{gathered}
\ddot{x}-2 n \dot{y}+\left(1-\alpha n^{2}\right) x=0, \\
\ddot{y}+2 n \dot{x}+y=0,
\end{gathered}
$$

with Jacobi integral

$$
\left(\dot{x}^{2}+\dot{y}^{2}\right)+\left(1-\alpha n^{2}\right) x^{2}+y^{2}=C .
$$

In order for the solution to Eq. (8) to be stable, the eigenvalues of the secular equation must be purely imaginary. It is readily verified that this requires that

$$
1+\frac{(4-\alpha)}{2} n^{2} \pm \frac{n}{2}\left[16+(4-\alpha)^{2} n^{2}\right]^{1 / 2} \geq 0
$$

Taking the negative sign (as the inequality is always satisfied for the positive sign), this can be solved for $n$ to yield

$$
n \leq \alpha^{-1 / 2} \simeq 0.58 \text { for } \alpha=3 .
$$

Since smaller values of $n$ correspond to more compact clusters (relative to the local galactic tidal force) this makes sense; the more compact clusters should be more stable against the galactic tidal interaction than the less compact ones. This simple theory predicts a definite upper limit to the characteristic number $n$. Since real clusters do not have square-law potentials, and the galactic tidal term will be modified due to the non-Keplerian motion of the cluster around the galaxy, and since real clusters, particularly globulars, move on elliptical orbits, the actual value of $n$ must be somewhat less than this. This "Roche limit" for clusters is related to the lower limit on cluster density of $\sim 10^{-1} M_{\odot} \mathrm{pc}^{-3}$ given by Mihalas and Routly (1968, p. 239). Their estimate is based on a force-balancing argument, while this estimate is due to an actual instability of the equations of motion.

\section{STABILITY ANALYSIS}

To analyze the orbits for stability and for the possible existence of a "pseudointegral" other than the Jacobi integral, the method of surface of section was employed. This has been described by many authors (Poincaré 

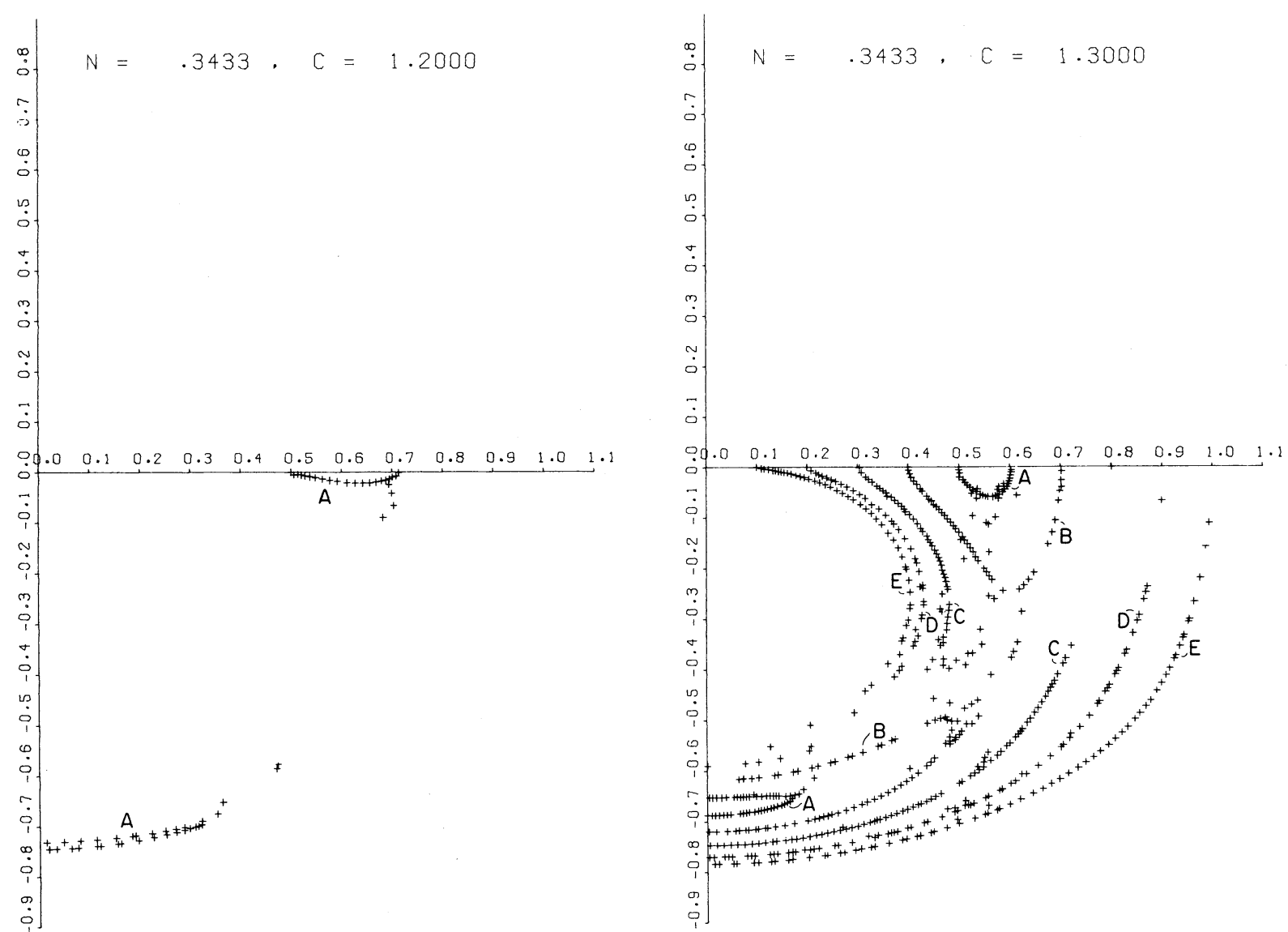

FIG. 1. Sample surface of section, $C=1.2$. Retrograde motion only. FiG. 2. Sample surface of section, $C=1.3$. Retrograde motion only.

1892; Hénon and Heiles 1964; see Jefferys 1974 for a list of references). The surface of section used here is similar to that in the last-named reference, and the reader is referred to it for more details.

In order to plot a surface of section on a flat sheet of paper, the problem must have two degrees of freedom. Methods have been developed to study systems with three degrees of freedom (Froeschlé 1970, 1972). Equations (1) in fact do describe a system of three degrees of freedom; however, for this first study it was thought best to consider a planar problem by setting $z$ $=0$, as Froeschlé's methods are considerably more complex to interpret. Thus, we will still be able to investigate direct and retrograde orbits in the cluster and to form some conclusions about them.

The equations of motion (with $z=0$ ) are symmetric about both the $x$ and $y$ axes; that is, if an orbit is seen reflected in either axis, and is traversed in the opposite sense, another orbit is obtained which satisfies the equations of motion. These symmetries are made use of in this work.

For the surface of section, we plot the position of a star in its orbit at each of the times that $\dot{r}=0$; thus, we plot pericenters and apocenters of the star's orbit, relative to the cluster center. If a second pseudointegral exists for the motion, the points will line up on several closed curves; otherwise, they will form a scattered set, and may escape the cluster. Hénon and Heiles call the latter case "ergodic." To reduce computing time and to distinguish direct from retrograde orbits, use has been made of the two symmetries in the problem mentioned above. If the pericenter of a direct orbit is at $(x, y)$, it has been plotted at $(|x|,|y|)$ while for retrograde orbits it has been plotted at $(|x|,-|y|)$. Thus, all points plotted appear in the right half-plane, reflected if necessary, while direct orbits appear in the upper half-plane, and retrograde orbits appear in the lower half-plane. This is very similar to the scheme used by Jefferys (1974).

Figures 1-10 show the computed surfaces of section for various values of the Jacobi constant $C$, adopting $\alpha$ $=3$ and $n=0.3433$. The larger values of $C$ correspond to the lower energies, and hence to orbits which are more strongly bound. Each figure contains several orbits, and the surfaces of section corresponding to each orbit have been distinguished by letters. Note the following important features: (a) For low to intermediate energies, all orbits are bounded by the zero-velocity surface. In addition, a pseudointegral appears to exist in each case. 




FIG. 3. Sample surface of section, $C=1 . \dot{4}$. Retrograde motion only.

(b) There is a marked difference in the behavior of the invariant curves for direct and retrograde orbits. (c) Just before the opening of the Roche lobe (for $C=1.7677$ ), the pseudointegral abruptly disappears for most of the direct orbits, while remaining for the retrograde orbits. (d) Shortly after the opening of the Roche lobe, nearly all direct orbits escape the system, while the retrograde orbits persist to much higher energies (lower values of $C$ ). (e) Retrograde orbits can persist somewhat beyond the tidal limit at $x=1$. (f) The cluster is strongly "stretched" in the $x$ direction, becoming roughly a prolate spheroid with a ratio of minor to major axes of roughly 0.8 , for these values of $n$ and $\alpha$.

Indeed, this value of the ellipticity is readily understood by considering Eq. (9), which predicts that the zero-velocity curves $\left(\dot{x}^{2}+\dot{y}^{2}=0\right)$ for the linearized case satisfy

$$
\left(1-\alpha n^{2}\right) x^{2}+y^{2}=C,
$$

which forms a family of ellipses with axis ratio

$$
E=\left(1-\alpha n^{2}\right)^{1 / 2} \cong 0.80 \text {. }
$$

This holds in the inner parts of the cluster, while in the outer parts, the galactic tidal field exerts a larger influ-

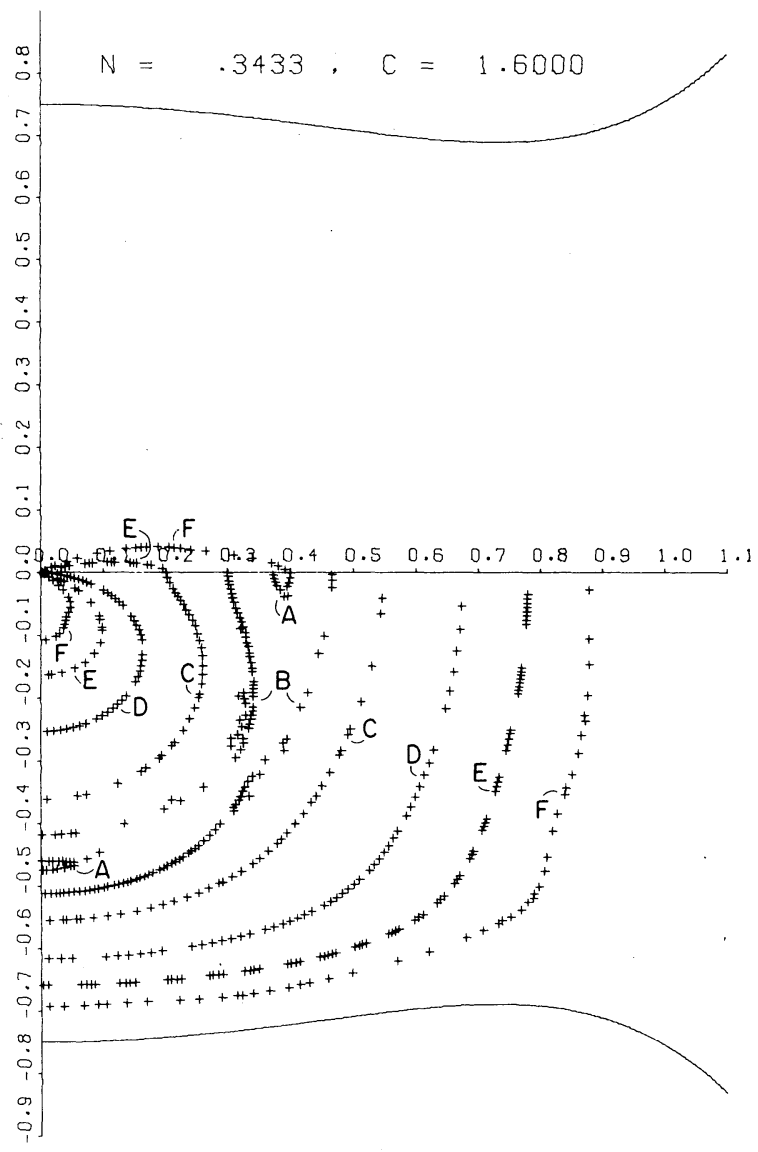

FIG. 4. Sample surface of section, $C=1.6$. Retrograde motion dominates.

ence and tends to elongate the cluster more, until the zero-velocity curves open up and allow stars to escape the cluster.

Note that the direction of the cluster's elongation is opposite to the direction of the long axis of the epicyclic orbit for field stars. This can be confirmed by solving Eqs. (8), less the terms $x$ and $y$ due to the cluster itself which appear in the first and second equations, respectively. One obtains for the ratio between the radial and tangential axes of the epicyclic orbit the value (4 $\alpha)^{1 / 2} / 2$, which, when the expression for $\alpha$ is inserted, equals $[-B /(A-B)]^{1 / 2}$, the usual value expressed in terms of Oort's constants.

At this point, it is important to mention a classic paper of Jeans (1922), in which he studied the effects of the other stars in a galaxy on the shape of a galactic cluster. $\mathrm{He}$ found two effects, one of which is a flattening of the cluster in the direction of its motion among the field stars due to a kind of "dynamical friction." The second effect, and the one relevant to the present discussion, was a tendency for a cluster far from the center of the galaxy to become elongated under the galactic force. This effect has some similarities to the effects discussed in this paper, but there are some important differences as well. 


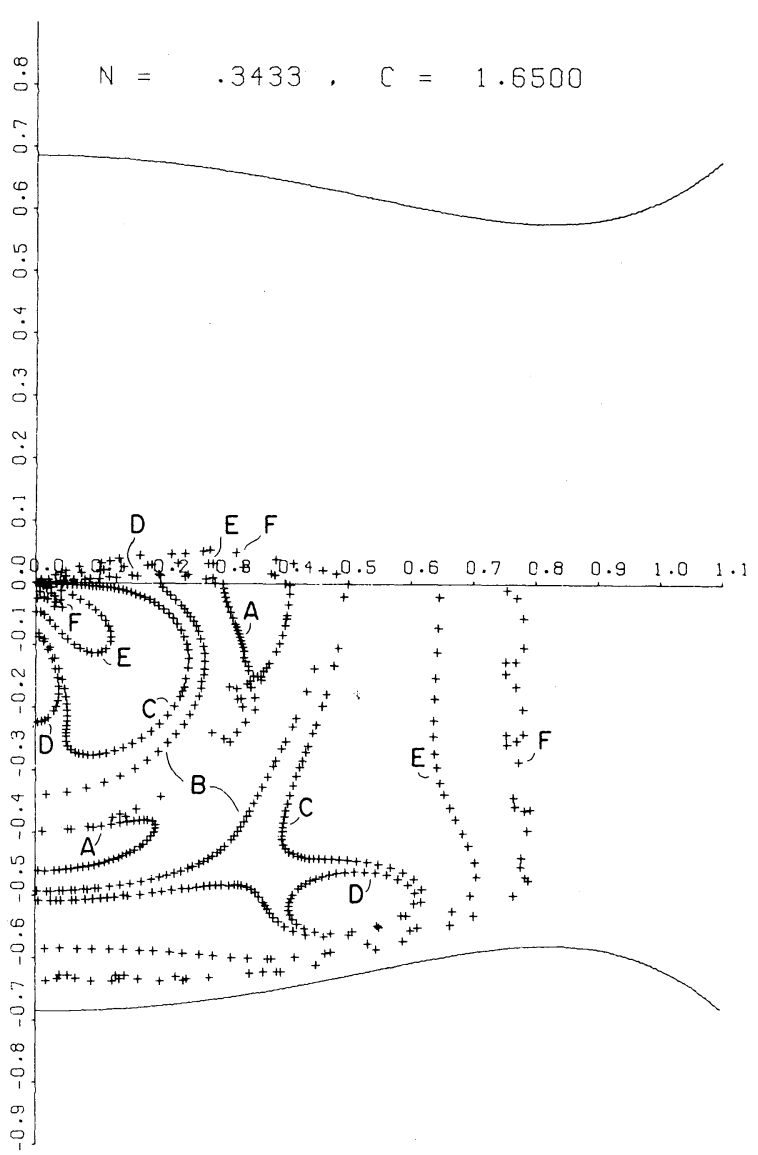

Fig. 5. Sample surface of section, $C=1.65$. Retrograde motion dominates. Note strong resonance effects.

In the first place, Jeans' equations of motion did not include the effects of galactic rotation, since they were formulated in the context of the Kapteyn universe. He did not consider the effects of the Coriolis terms, which in the present model are responsible for coupling the $x$ and $y$ equations, and play a very important rôle. In addition, the elongation he found was due to an instability which causes the cluster to expand along one axis at an exponentially growing rate. It is not a quasistationary effect such as is discussed here.

In some of the surfaces of section, nonlinear effects are particularly prominent. Notable are the surfaces of section for $C=1.6-1.8$, where invariant curves are severely distorted, resonances exert important influences, and (for $C=1.7677$ ), orbits which are bounded by the zero-velocity curves nevertheless become "unstable" in an ergodic sense (Hénon and Heiles 1964).

The stability properties of the orbits are summarized in Fig. 11, an Hénon stability diagram. On this diagram, the lightly shaded region represents stable orbits; the heavily shaded region, the region forbidden to all orbits by the Jacobi integral. A point $(C, y)$ in the diagram represents an orbit with Jacobi constant $C$, and initial

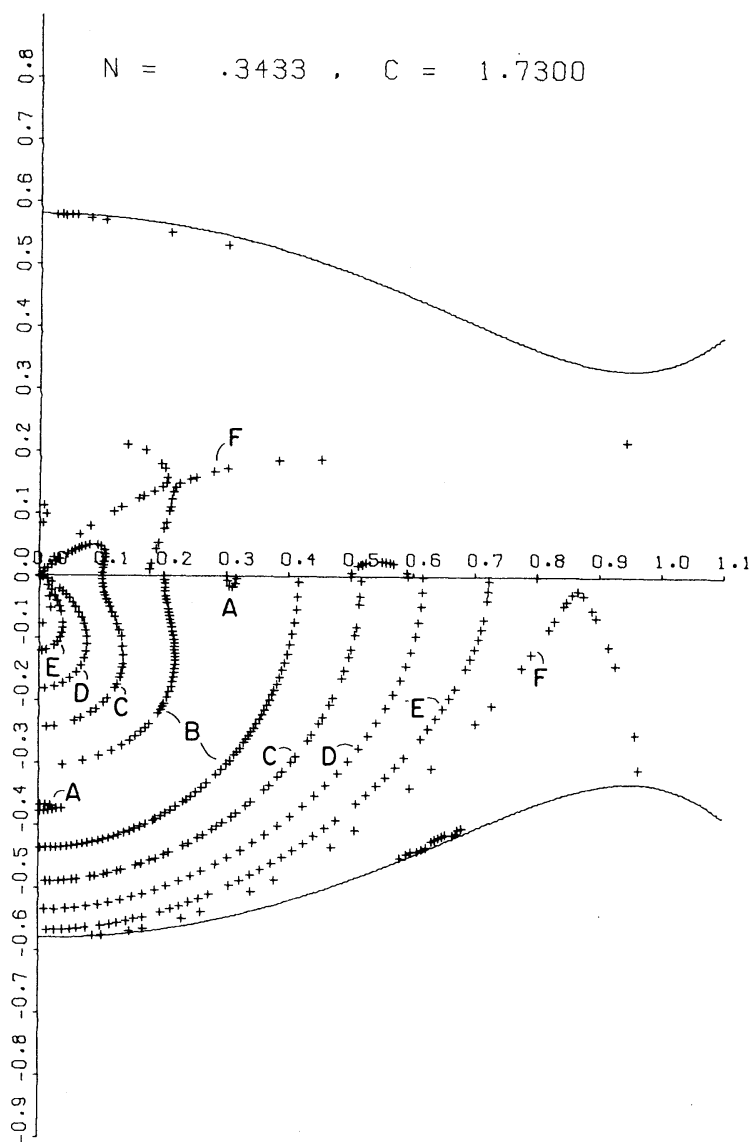

FIG. 6. Sample surface of section, $C=1.73$. Retrograde motion dominates. Direct orbits are nearly all escapers.

conditions $x=\dot{y}=0, y$ given on the abscissa, and $\dot{x}$ given by solving Eq. (3), with the positive sign of the square root being taken if $y<0$ and the negative sign otherwise.

Two notable features of this diagram are (a) the excess of retrograde orbits, and the fact that retrograde orbits are stable to much greater distances than the direct ones. This suggests that one would expect the outer regions of a cluster to exhibit retrograde motion, but not the inner regions. Also, (b) there is a notch-like region near $C=$ 1.6 devoid of stable retrograde orbits. This is apparently due to low-order resonances, and demonstrates that resonance phenomena cannot be ignored in constructing realistic cluster models. Taken together these two effects show that the estimates of the "tidal radius" of clusters given in the literature (e.g., King 1962, 1966; Peterson and King 1975) are probably not precise, although they do give order-of-magnitude estimates. Indeed, Keenan and Innanen (1975) have remarked that their model clusters seem somewhat larger than the tidal radius given by King's formula, and this is consistent with the present work.

To summarize these results for the circular case, the 




Fig. 7. Sample surface of section, $C=1.7677$. Roche lobe closes. $\Lambda$ large proportion of the direct part of the phase space is occupied by "ergodic" orbits.

model predicts (a) the existence of a second pseudointegral; (b) greater stability for the retrograde orbits, as found by Keenan, Innanen, and House; and (c) a strong tidal effect, tending to "stretch" the cluster into a prolate form.

\section{CLUSTERS HAVING ORBITS OF HIGH ECCENTRICITY}

The model discussed in Sec. II is applicable to galactic clusters, but is not at all realistic when applied to globulars because of the large eccentricity of their orbits around the galaxy. To gain some insight into what might happen in this case, let us consider the opposite extreme, that is, a cluster whose orbit is rectilinear, passing straight through the galactic center. It is assumed that the cluster avoids encounters with any supermassive black holes that may be lurking there!

In the inner parts of a cluster, the motion of a star is governed primarily by a harmonic oscillator-like potential obtained by expanding Eq. (4) in powers of $r / r_{0}$ and dropping terms of order higher than 2 . There are no Coriolis or centrifugal force terms. Therefore, for this



Fig. 8. Sample surface of section, $C=1.8$. Most orbits stable.

discussion we ignore the outer parts of the cluster, and write

$$
\begin{aligned}
& \ddot{x}=\partial V / \partial x, \\
& \ddot{y}=\partial V / \partial y, \\
& \ddot{z}=\partial V / \partial z,
\end{aligned}
$$

where

$$
V=1-\frac{1}{2}\left(x^{2}+y^{2}+z^{2}\right)+V_{G},
$$

$V_{G}$ being the differential potential due to the galaxy, which produces a periodically varying tidal force on the cluster as the cluster moves on its orbit. Let the cluster's orbit be a straight line passing through the galactic center, and let the $x$ axis be aligned along it.

The force on a star due to the galaxy varies with the distance from the galactic center. It rises to a maximum some distance from the center, then falls again. The force is primarily directed along the $x$ axis, with much smaller components in $y$ and $z$ (particularly in the parts of the galaxy where most globulars are found). Since the force is directed inwards, the effect in the inner regions of the galaxy is for the stars farther out to be accelerated more towards the galactic center than the stars slightly closer 


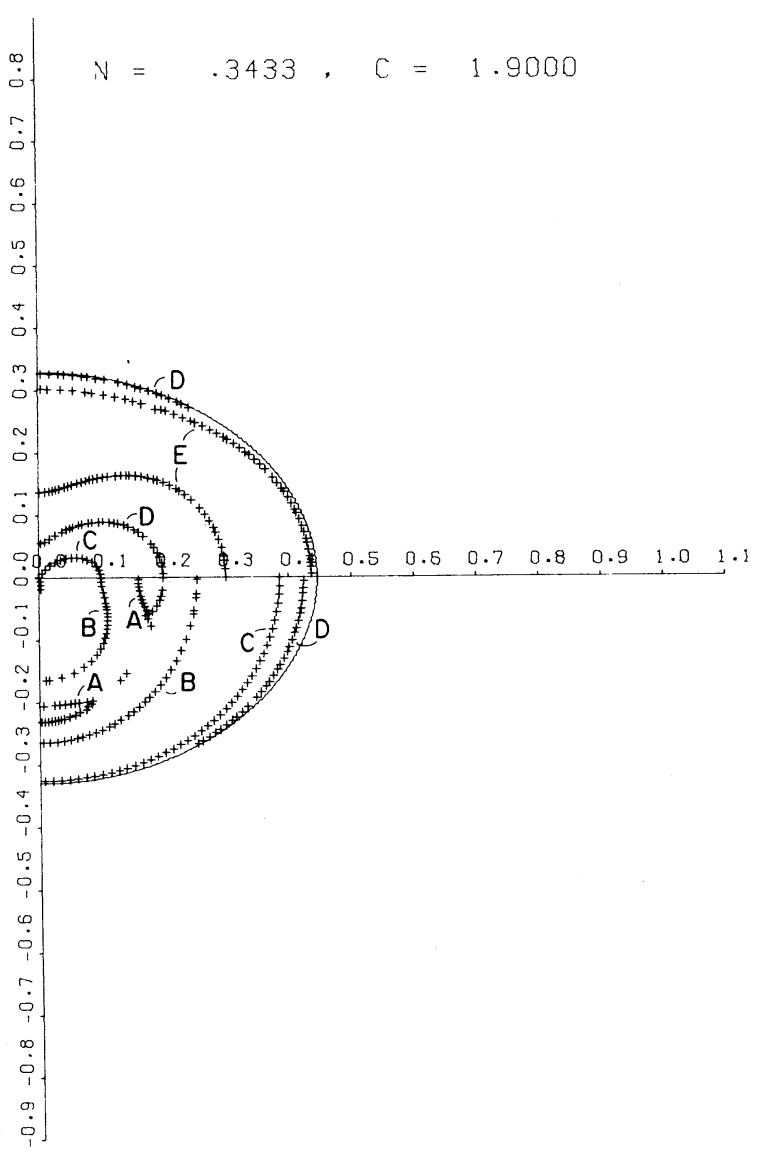

Fig. 9. Sample surface of section, $C=1.9$. All orbits stable.

in, thus tending to compress the cluster in the $x$ direction. In the outer regions of the galaxy, the effect is exactly opposite.

This effect can be effectively modeled as a variable spring constant in the $x$ component of Eqs. (14), such that the spring constant is greatest when the cluster is at the galactic center, and least at the extreme ends of the cluster's orbit. The equation of motion in $x$ therefore becomes

$$
\ddot{x}=-[1+\epsilon \psi(\theta)] x,
$$

where $\theta$, a linear function of time, is 0 or $\pi$ at the galactic center and $\pi / 2$ or $3 \pi / 2$ at the extreme ends of the orbit, and $\psi(\theta)$ is a periodic function having its maximum for $\theta=0, \pi$ and its minimum for $\theta=\pi / 2,3 \pi / 2$. The coefficient of the galactic tidal force, $\epsilon$, is assumed small compared to the self-gravity of the cluster.

The simplest model would be to take $\psi(\theta)=\cos \theta$, ignoring all but the fundamental term in the Fourier expansion of $\psi$, and absorbing any constant into the unperturbed harmonic oscillator. This approximation completely ignores any "gravitational shock" which may affect the cluster as it passes through the galactic plane (Keenan and Innanen 1975). Such transient effects

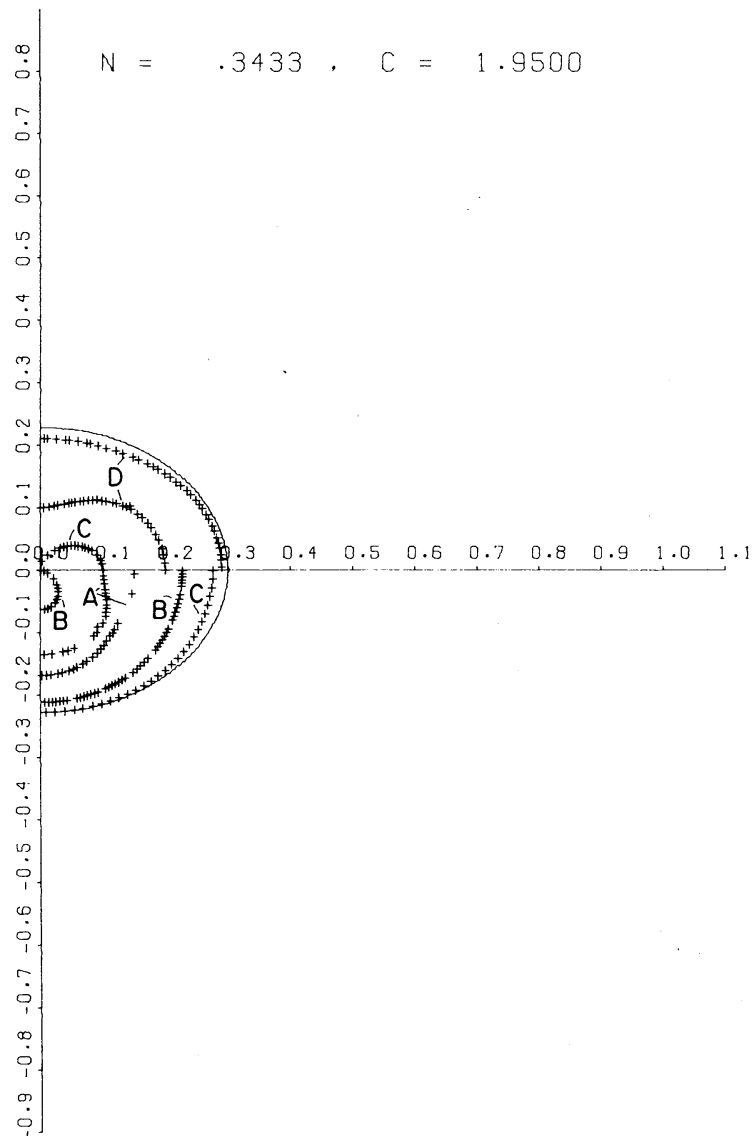

FIG. 10. Sample surface of section, $C=1.95$. All orbits stable

would be due to a superposition of very high harmonics in the perturbing force, and would best be treated without resorting to a Fourier expansion. It is possible that they would substantially alter the subsequent behavior of the cluster, but this possibility remains to be investigated.

Letting $\dot{\theta}=\omega$, the frequency of the cluster's oscillation in its orbit through the galaxy, Eqs. (16) can be derived from a Hamiltonian:

$$
\begin{aligned}
& F=\frac{1}{2}\left(x^{2}+X^{2}\right)+\frac{\epsilon}{2} x^{2} \cos \theta+\omega \theta, \\
& X=\dot{x}=\partial F / \partial X, \dot{\theta}=\partial F / \partial \theta \\
& \ddot{x}=\dot{X}=-\partial F / \partial x, \dot{\theta}=-\partial F / \partial \theta .
\end{aligned}
$$

Making the canonical transformation

$$
\begin{aligned}
& x=(2 K)^{1 / 2} \cos k, \\
& X=(2 K)^{1 / 2} \sin k,
\end{aligned}
$$

we have

$$
\begin{aligned}
F=K+\omega \theta+ & \frac{\epsilon}{8} K[2 \cos \theta \\
& +\cos (\theta+2 k)+\cos (\theta-2 k)],
\end{aligned}
$$




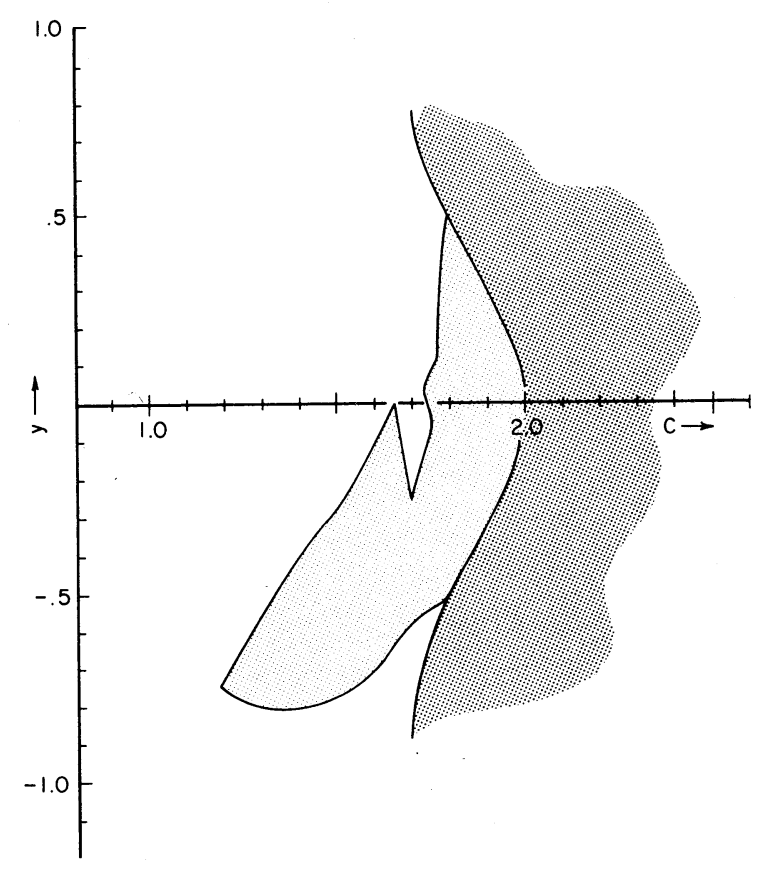

FIG. 11. Hénon stability diagram for cluster model of Sec. II.

where

$$
\begin{aligned}
\dot{k} & =\partial F / \partial K, \\
\dot{K} & =-\partial F / \partial k \\
& =-\frac{\epsilon}{8} K[-2 \sin (\theta+2 k)+2 \sin (\theta-2 k)] \\
& =\frac{\epsilon}{4} K[\sin (\theta+2 k)-\sin (\theta-2 k)] .
\end{aligned}
$$

Using the method of averaging, by approximating $K, k$, $\theta$ by $K^{*}, k^{*}, \theta^{*}$ obtained by ignoring the term in $\epsilon$ from Eq. (20), we find to first order,

$$
\begin{aligned}
K=K^{*}\left[1+\frac{\epsilon}{4}\left(\frac{-\cos \left(\theta^{*}+2 k^{*}\right)}{\omega+2}+\right.\right. \\
\left.\frac{\cos \left(\theta^{*}-2 k^{*}\right)}{\omega-2}\right] .
\end{aligned}
$$

Now consider the effect of this on the extent of the cluster in the $x$ direction. This will be seen in those stars for which $x$ is a maximum, that is, for values of $k^{*} \approx k$ $=0, \pi$. For this value, Eq. (23) becomes

$$
\begin{aligned}
K & =K^{*}\left[1+\frac{\epsilon}{4}\left(\frac{-\cos \theta^{*}}{\omega+2}+\frac{\cos \theta^{*}}{\omega-2}\right)\right] \\
& =K^{*}\left(1+\frac{\epsilon \cos \theta^{*}}{\omega^{2}-4}\right) .
\end{aligned}
$$

For real clusters, we expect the crossing time in the cluster to be considerably less than the period of the cluster around the galaxy-otherwise the cluster would not be bound against galactic tidal forces; therefore $\omega<$ 1 and $K$ has a minimum for $\theta^{*}=0, \pi$ at the center of the galaxy, and a maximum for $\theta^{*}=\pi / 2,3 \pi / 2$, at the extreme ends of the cluster's orbit. At the same time, the $y$ and $z$ motion, being effectively decoupled from the $x$ motion (except through mutual encounters), would be relatively unaffected. Thus, the model predicts that in the outer parts of the galaxy, the cluster would be stretched into a prolate form along the $x$ axis, while in the interior parts of the galaxy, it would assume an oblate form with its short axis pointing towards the galactic center. The exact point in the rectilinear orbit where the cluster would switch between oblate and prolate form would depend on the details of the energy balance between the three degrees of freedom $(x, y$, and $z)$ in the cluster, and on the details of the cluster's orbit. There should, however, be an overall tendency towards one form or the other in different regions of the galaxy.

\section{OBSERVED INCLINATIONS OF OBJECTS ON THE GALAXY}

The results of Sec. II show that there should be a tendency for galactic clusters, and by implication other extended objects moving in circular orbits around the galaxy, to be stretched into a prolate form with the long axis pointing towards the galactic center. Is there any evidence of such an effect? The answer is that there is some evidence. in the literature, but that it is far from conclusive. The results of Hopper and Disney (1974, 1975) show that alignment with the galactic plane is found for interstellar dust clouds, but it is not possible to distinguish between a prolate form such as is predicted in this study, and the oblate form suggested by their mechanism, for objects in the galactic plane. It is possible that both mechanisms are operative. One point which favors the prolate hypothesis for dust clouds is the fact that the alignment is more pronounced for $l^{\mathrm{II}} \sim 90^{\circ}$, since at such longitudes a prolate form would present its maximum extent to our view. [The model of Sec. I of this paper can be applied to dust clouds if pressure and dust-grain collisions are ignored (see Hopper and Disney 1974, 1975). However, it is questionable that the results of Melnick and Harwit can be explained in this way.]

The orientation of objects which were seen significantly above or below the galactic plane ought to depend on longitude, if the prolate hypothesis is correct. For, if an object is at $l^{\mathrm{II}} \sim 180^{\circ}$ and has a high galactic latitude, it would be seen "from the underside." If it is a prolate object, with its long axis pointing towards the galactic center, its inclination, seen in projection, should be near $90^{\circ}$. At galactic longitudes near $90^{\circ}$ or $270^{\circ}$, on the other hand, parallelism with the galactic plane should obtain, with intermediate values (depending on latitude) at intermediate longitudes. Shapley (1930) remarks that the galactic clusters in the Cassiopeia-Perseus region, which include several having rather large galactic latitude, exhibit a larger mean inclination to the galactic plane $\left(55^{\circ}-60^{\circ}\right)$ than elsewhere. On the other hand, the 
TABLE I. Globular cluster distributions taken from Shapley's data.

\begin{tabular}{lccc}
\hline & $0 \leqslant|i| \leqslant 30^{\circ}$ & $30^{\circ} \leqslant i \leqslant 60^{\circ}$ & $60^{\circ} \leqslant i \leqslant 90^{\circ}$ \\
\hline$\left|b^{\mathbf{I I}}\right| \leqslant 30^{\circ}$ & 13.5 & 4.5 & 8 \\
$\left|b^{\mathbf{I I}}\right| \geqslant 30^{\circ}$ & 2 & 3 & 6 \\
\hline
\end{tabular}

high latitude $\left(>10^{\circ}\right)$ objects in Shapley's catalog show no such correlation.

Shapley's catalog of galactic clusters definitely exhibits a longitude dependence in the cluster orientations, as he notes. There is a strong tendency for the clusters in the sectors perpendicular to the direction to the galactic center (longitudes $45^{\circ}-135^{\circ}$ and $225^{\circ}-315^{\circ}$ ) to have large inclinations of their long axes to the galactic plane. In fact, of 22 such clusters, only two have inclinations $\leq 30^{\circ}$. Assuming a uniform distribution of inclinations, the $\chi^{2}$ test gives a probability of this occurring by chance of less than $2 \%$. On the other hand, in the sectors $135^{\circ}-225^{\circ}$ and $315^{\circ}-45^{\circ}$, there are 16 clusters with inclinations $\leq 30^{\circ}$ and 20 clusters of $>30^{\circ}$; one would have expected 12 and 24 , respectively. The probability of this occurring by chance is about $15 \%$. Thus, there is a strong tendency towards perpendicularity in the sectors $45^{\circ}-135^{\circ}$ and $225^{\circ}-315^{\circ}$, and a weaker tendency towards parallelism towards the galactic center and anticenter. However, these tendencies are the opposite of what would be expected for prolate objects pointing towards the galactic center, at least in the longitude ranges $45^{\circ}-135^{\circ}, 225^{\circ}-315^{\circ}$. The model of Sec. II is apparently too simple to explain these data.

Some other interesting statistics can be obtained from Shapley's study of globular clusters. He has measured and tabulated the inclinations of the observed long axes of 37 globular clusters relative to the galactic circle. Let us ask what we ought to observe, if the model of Sec. III of this paper approximates the actual situation.

Globulars tend to be concentrated towards the galactic center, and according to this model, the ones closest to the galactic center should tend to align with it, while the ones farther away from the center should tend to align in the perpendicular direction. Looking along our line of sight we should see a mixture of the two populations, with relatively few clusters having intermediate values of the inclination. Clusters observed at higher latitudes should have a greater proportion of large to small inclinations than those observed at lower latitudes, since they are systematically farther from the galactic center, and are far from the galactic plane.

The actual distribution, taken from Shapley's data (except that $l^{\mathrm{II}}, b^{\mathrm{II}}$ has been used instead of $l^{1}, b^{\mathrm{I}}$ ), is shown in Table I (one cluster with $i=30^{\circ}$ has been shared between two bins). Note the relative infrequency with which intermediate values of $i$ occur. The $\chi^{2}$ distribution has been used to investigate the probability that these distributions occurred by chance, assuming as a
TABLE II. Statistics for globular cluster model.

\begin{tabular}{lccc}
\hline & $0^{\circ} \leqslant i \leqslant 30^{\circ}$ & $30^{\circ} \leqslant i \leqslant 60^{\circ}$ & $60^{\circ} \leqslant i \leqslant 90^{\circ}$ \\
\hline$\left|b^{\mathrm{II}}\right| \leqslant 30^{\circ}$ & 13.6 & 5.3 & 7.7 \\
$\left|b^{\mathrm{II}}\right| \geqslant 30^{\circ}$ & 1.8 & 0.9 & 7.8 \\
\hline
\end{tabular}

null hypothesis a uniform angular distribution. For $\left|b^{\text {II }}\right|$ $\geq 30^{\circ}$, the $\chi^{2}$ test gives a $25 \%$ probability - not very interesting, although the trend of the data is as predicted. For $\left|b^{11}\right| \leq 30^{\circ}$, the probability is $<10 \%$-more interesting, but not conclusive. Unfortunately, the data are sparse, so the statistics are not stronger. Furthermore, the mean error of one of Shapley's measured inclinations is roughly $\pm 30^{\circ}$, which degrades the quality of the statistics.

Nevertheless, it would be interesting to see if the prolate-oblate hypothesis can duplicate these figures. A simple model was employed. Clusters were selected at random positions within the galaxy by choosing points from a trivariate Gaussian distribution

$$
f(x, y, z)=(2 \pi)^{-3 / 2} \sigma^{-3} \exp \left[-\left(x^{2}+y^{2}+z^{2}\right) / 2 \sigma^{2}\right],
$$

where $\sigma=5 \mathrm{kpc}$ was chosen. The Sun was located at the point $(10,0,0) \mathrm{kpc}$, and clusters within $\pm 5^{\circ}$ of the galactic plane were excluded as being probably obscured. The alignment of the observed long axis of the cluster with the galactic plane was calculated on the assumption that within a certain ellipsoid of revolution,

$$
x^{2}+y^{2}+z^{2} / s^{2} \leq t^{2},
$$

the cluster would be oblate and perpendicular to the axis to the galactic center; outside, it would be prolate and parallel to that axis. Various values of $s$ and $t$ were run. The best fit was obtained for $s=0.5, t=11.3$ (for which $\chi^{2}=2.12$ ). The statistics for this model are shown in Table II.

The "squashing factor" $s$ was introduced into the model to account for the flattening of the galaxy, and hence a general tendency for the point of switchover between prolate and oblate forms to be closer to the galactic center when the cluster is moving out of the galactic plane than when it is moving near the plane. Since this model is very crude, little significance should be ascribed to the actual values of $s$ and $t$.

\section{SUMMARY}

Systematic alignment of the long axes of extended objects with and against the direction of the galactic plane has been suspected for several groups of objects. Theoretical models for two limiting cases of the eccentricity of a cluster's orbit in the galaxy predict a strong effect from the differential galactic tidal force on the shape of such an object, which can either stretch it into a prolate shape or squash it into an oblate shape, de- 
pending on the circumstances and location of the cluster. Other effects (differential $z$ force, random effects) are also probably important.

In the case where the orbit of a cluster is circular, the existence of a second pseudointegral of the motion (other than the energy) is confirmed for the planar case. One effect of this integral is to engender a considerably greater stability of retrograde over direct orbits.

Some support for the hypothesis that tidal forces are important in shaping clusters is given by several observational studies. However, the evidence is inconclusive and not entirely favorable, and further work needs to be done to confirm or deny this hypothesis.

\section{ACKNOWLEDGMENTS}

The author wishes to thank Dr. I. King for several stimulating discussions, and Dr. G. de Vaucouleurs for help in locating observational materials.

Note added in proof: I should like to thank Dr. Ivan King for several important observations. The potential $\sinh ^{-1}\left(r / r_{0}\right) / r$ is considerably more realistic than the Plummer potential and is to be preferred. Fortunately, recalculations of the galactic cluster model using this potential, while differing in detail, do not appear to alter the main conclusions of the paper. Secondly, the originally chosen value of $r_{0}$ for globulars, used to compute the parameter $n$, was too big, an error also noted by the referee. Since the model is inappropriate to globulars anyway, because of the assumed circular orbit, this is not too important, but the value given in the body of the paper has now been revised on the basis that it is computed at perigalacticon, where the tidal forces would be strongest. Finally, I had overlooked an important paper by B. Bok (1934, Harvard Circ. No. 384), in which the instability of an equation similar to Eqs. (8) is discussed.

\section{REFERENCES}

Froeschlé, C. (1970). Astron. Astrophys. 4, 115.

Froeshclé, C. (1972). Astron. Astrophys. 16, 172.

Griffin, R. (1972). Observatory 92, 29.

Harding, G. A. (1965). R. Obs. Bull. No. 99.

Hénon, M. (1969a). Astron. Astrophys. 1, 223.

Hénon, M. (1969b). Astron. Astrophys. 2, 151.

Hénon, M. (1970). Astron. Astrophys. 9, 24.

Hénon, M., and Heiles, C. (1964). Astron. J. 69, 73.

Hopper, P. B., and Disney, M. J. (1974). Mon. Not. R. Astron. Soc. $168,639$.

Hopper, P. B., and Disney, M. J. (1975). Mon. Not. R. Astron. Soc. $170,177$.

Hunter, R. B. (1967a). Mon. Not. R. Astron. Soc. 136, 245.

Hunter, R. B. (1967b). Mon. Not. R. Astron. Soc. 136, 267.

Jackson, J. (1913). Mon. Not. R. Astron. Soc. 74, 62.

Jeans, J. H. (1922). Mon. Not. R. Astron. Soc. 82, 132.

Jefferys, W. H. (1974). Astron. J. 79, 710.

Johnson, H. M. (1952). Astrophys, J. 115, 124.

Keenan, D. W., and Innanen, K. A. (1975). Astron. J. 80, 290.

Keenan, D. W., Innanen, K. A., and House, F. C. (1973). Astron. J. 78, 173.

King, I. (1962). Astron. J. 67, 471.

King, I. (1966). Astron. J. 71, 64.

Lynds, B. T. (1962). Astrophys. J. Suppl. 15, 97.

Melnick, G., and Harwit, M. (1975). Mon. Not. R. Astron. Soc. 171, 441.

Mihalas, D., and Routly, P. M. (1968). Galactic Astronomy (Freeman, San Francisco).

Peterson, C. J., and King, I. R. (1975). Astron. J. 80, 427.

Poincaré, H. (1892). Les Méthodes Nouvelles de la Mécanique Céleste (Gauthier-Villars, Paris).

Shapley, H. (1930). Star Clusters, Harvard Obs. Monograph No. 2 (McGraw-Hill, New York). 